\title{
Erratum to: Simultaneous determination of NNK and its seven metabolites in rabbit blood by hydrophilic interaction liquid chromatography-tandem mass spectrometry
}

\author{
Hailei Lang • Sheng Wang • Qidong Zhang • \\ Beibei Zhao - Lei Wang • Baojun Cao • Juan Wang • \\ Jian Mao $\cdot$ Jianxun Zhang
}

Received: 2 April 2013 / Accepted: 2 April 2013 /Published online: 19 May 2013

(C) Springer-Verlag Berlin Heidelberg 2013

Erratum to: Analytical and Bioanalytical Chemistry DOI 10.1007/s00216-012-6641-z

The authors would like to call the reader's attention to the fact that unfortunately there was a mistake in Table 3 of this contribution. Please find the correct Table 3 below:

The online version of the original article can be found at http://dx.doi.org/ $10.1007 / \mathrm{s} 00216-012-6641-\mathrm{z}$

H. Lang $\cdot$ S. Wang $\cdot$ Q. Zhang $\cdot$ L. Wang $\cdot$ B. Cao $\cdot$ J. Mao $\cdot$

J. Zhang $(\bowtie)$

Zhengzhou Tobacco Research Institute of CNTC,

Zhengzhou 450001, China

e-mail: jxzh258@163.com

B. Zhao

Quality Supervision and Test Station of the Guangxi Tobacco

Monopoly Administration, Nanning 530022, China

J. Wang

Quality Supervision and Test Station of Hainan Tobacco

Corporation, Haikou 571100, China
Table 3 Recovery and RSD of NNK and its metabolites in rabbits plasma

\begin{tabular}{|c|c|c|c|}
\hline Analyte & $\begin{array}{l}\text { Concentration } \\
\left(\mathrm{ng} \mathrm{mL} \mathrm{mL}^{-1}\right)\end{array}$ & $\begin{array}{l}\text { Mean recovery } \\
(\%, n=5)\end{array}$ & $\begin{array}{l}\text { RSD } \\
(\%, n=5)\end{array}$ \\
\hline \multirow[t]{3}{*}{ NNK } & 50 & 107.0 & 0.85 \\
\hline & 10 & 116.3 & 5.46 \\
\hline & 2 & 78.9 & 1.50 \\
\hline \multirow[t]{3}{*}{ HPB } & 25 & 101.7 & 1.99 \\
\hline & 5 & 112.9 & 5.92 \\
\hline & 1 & 76.9 & 1.54 \\
\hline \multirow[t]{3}{*}{ NNAL } & 50 & 101.4 & 1.48 \\
\hline & 10 & 99.8 & 1.56 \\
\hline & 2 & 97.6 & 3.15 \\
\hline \multirow[t]{3}{*}{ PBD } & 25 & 93.6 & 0.86 \\
\hline & 5 & 107.3 & 6.52 \\
\hline & 1 & 106.1 & 4.03 \\
\hline \multirow[t]{3}{*}{ NNK- $N$-Oxide } & 25 & 109.2 & 0.54 \\
\hline & 5 & 98.2 & 4.08 \\
\hline & 1 & 93.6 & 4.21 \\
\hline \multirow[t]{3}{*}{ NNAL- $N$-Oxide } & 25 & 107.0 & 4.02 \\
\hline & 5 & 104.4 & 3.03 \\
\hline & 1 & 102.3 & 4.23 \\
\hline \multirow[t]{3}{*}{ Keto acid } & 25 & 92.0 & 0.53 \\
\hline & 5 & 101.7 & 1.99 \\
\hline & 1 & 82.3 & 1.81 \\
\hline \multirow[t]{3}{*}{ Hydroxy acid } & 25 & 109.2 & 0.54 \\
\hline & 5 & 108.8 & 5.94 \\
\hline & 1 & 90.8 & 4.10 \\
\hline
\end{tabular}

